\title{
Research and Experimental Application of Family Holographic Projection Entertainment Equipment
}

\author{
Anzhuo $\mathrm{Li}^{1}$, Fan $\mathrm{Li}^{2}$ and Dayong $\mathrm{Ma}^{\mathrm{a}}$ \\ ${ }^{1}$ School of Design Art, Shenyang Aerospace University, Shenyang 110000, China \\ ${ }^{2}$ School of Design Art, Shenyang Aerospace University, Shenyang 110000, China
}

\begin{abstract}
This paper introduces a kind of family entertainment equipment based on holographic projection technology and optical principle. It can achieve 3D viewing of video effect without wearing any head display equipment, with a sense of immersion and substitution. The equipment is mainly composed of external stereo structure and internal specific source. The results can be used for family entertainment. In the process of using, it can be visualize, embodied and effectively reduce the damage of digital devices to users' eyes.
\end{abstract}

\section{Introduction}

In recent years, with the development of science and technology, digital entertainment products such as VR and AR have gradually entered into people's lives. But at present, most of VR and AR products are inseparable from the participation of smart phones, televisions, tablet computers and other hardware devices. It is still necessary to be able to experience by mean of an external equipment and not to achieve a full 3D naked eye experience. This shortcoming will bring physical harm to users, especially to children. However, with the help of the technology of holographic projection, the naked eye can be viewed 360 degrees in all directions. The biggest advantage is that it does not need to wear any equipment. This paper aims at the entertainment demand of ordinary users, the learning and cognitive needs of the young children and the entertainment experience needs of the cartoon enthusiasts, and develops a set of holographic projection entertainment products with full naked eyes.

\section{Function and principle}

\subsection{Equipment Function}

The family holographic projection entertainment equipment is mainly composed of two parts of the external stereoscopic structure and the internal specific source, and the external stereoscopic equipment converges the specific sheet source picture to the center. Making use of holographic projection technology to make two-dimensional audio-visual broadcasting become three-dimensional. Through this holographic rendering, the user's horizons can be planed and stereoscopic. Giving them a deep sense of immersion also greatly reduces the damage to the eyes of digital products, and thus has a better user experience.

\subsection{Fundamental principle}

Holographic projection technology is the technique of recording and reproducing the real three-dimensional image of the object by the principle of reflection, refraction and diffraction of light. The projected image is the intensity distribution of the Fraunhofer diffraction image produced by the pure phase hologram. In this paper, we use the iterative Fourier transform algorithm, which is the most commonly used in the process of simulating optical diffraction, and use Fourier lens to realize Fraunhofer diffraction of plane images. The schematic diagram is shown in Figure 1.

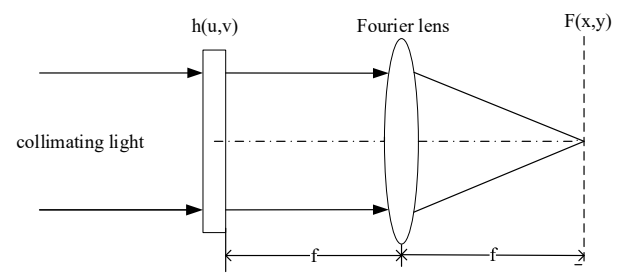

Figure 1. Schematic diagram of holographic projection.

Assuming that the hologram input formula is expressed by $\mathrm{h}(\mathrm{u}, \mathrm{v})=\exp \left(\mathrm{i} \varphi_{n}\right)$, when the collimating light source is irradiated, the complex amplitude distribution $\mathrm{F}(\mathrm{x}, \mathrm{y})$ of the Fourier lens is equal to the Fourier transformation of the hologram.

$$
\mathrm{F}(\mathrm{x}, \mathrm{y})=\mathfrak{L}\{\mathrm{h}(\mathrm{u}, \mathrm{v})\}
$$

$\mathrm{h}(\mathrm{u}, \mathrm{v})$ is the amplitude distribution, $\varphi$ is the phase function, and $\mathfrak{L}$ is Fourier transform.

\section{Structural analysis}

The structural design of the equipment is shown in Figure 2. The outer structure is embedded with a four pyramid in the cube border. The surface of the four pyramids is composed of four isosceles triangle with 70.5 equal angles

* Corresponding author: ${ }^{a} 381772091 @ q q . c o m$ 
at the top angle of 70.5 degrees, and the angle is 45 degrees from the ground.

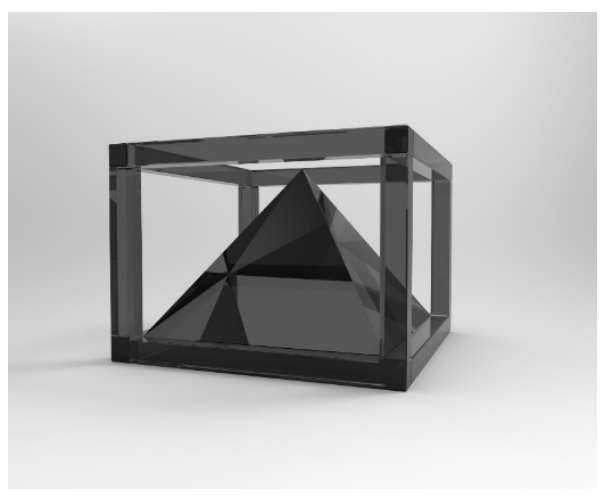

Figure 2. Overall schematic diagram of equipment.

The source of the equipment plays from the front, back, left, right four angles of the object, using video editing software to recombine the video into a video source file with four surfaces at the same time. When the equipment is used, each of the four pyramids reflects the image of the corresponding plane, and the video of each surface is synthesized into three-dimensional imaging by reflection, refraction and diffraction of light. Because the distance between the light and the eyes of different faces is different, the video will form a certain time difference between the users, and the stereoscopic image that is accepted has a deep sense. In brief, the holographic projection entertainment equipment is to process the captured video into four video sources (as shown in Figure 3). The four overlapping images which are reflected by the four pyramids are combined into a threedimensional picture, so that the original plane sense image has a three-dimensional sense.

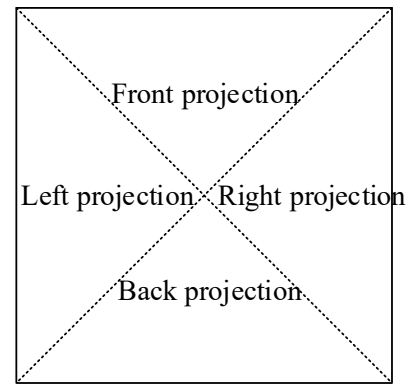

Figure 3. Projection video source distribution map.

Only when the bottom and waist length reach a certain proportion can the best playing effect be achieved. Based on certain experimental calculations and repeated argumentation, the optimal data obtained in this paper are as follows.

Bottom length $=$ screen width

The height of isosceles triangle $=$ bottom edge length $/ 0.5=$ bottom edge length $* 0.707$

Isosceles triangle oblique side length $=$ bottom edge length $/ 2 \cos 54$ degrees $75^{\prime}=$ bottom side length $* 0.866$

\section{Implementation results}

\subsection{Film production process}

In the production of film source, we mainly use 3D model software and later software to obtain holographic projection sources.

(1) Use 3D software such as Autodesk Maya or $3 \mathrm{ds}$ MAX to model the roles, and export the models in obj. format.

(2) Brush the weight for the role model, that is, adjust the influence of the skinned bones on the fixedpoint of the object.

(3) Use Autodesk MotionBuilder to create the connection relationship between the captured action and the bound bones, and map the data to the virtual skeleton of the role.

(4) Use 3D software such as 3ds MAX to create cameras at four angles of view around the role model, and maintain a fixed distance to follow the role model movement.

(5) The moving images are rendered, and the different images of the four cameras are presented, and the video is synthesized by the synthetic software to match the music according to a certain structure.

\subsection{System implementation}

Figure 4 are special film sources based on the process, which collect the real time images of the four sides of the object and processes them.

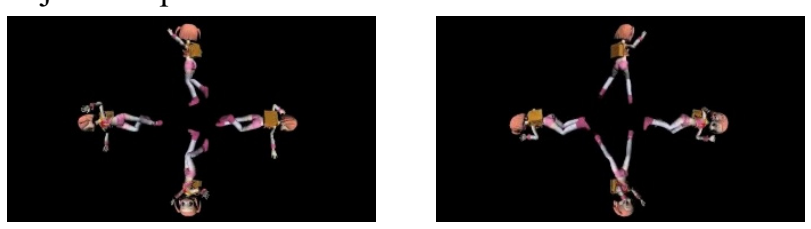

Figure 4.
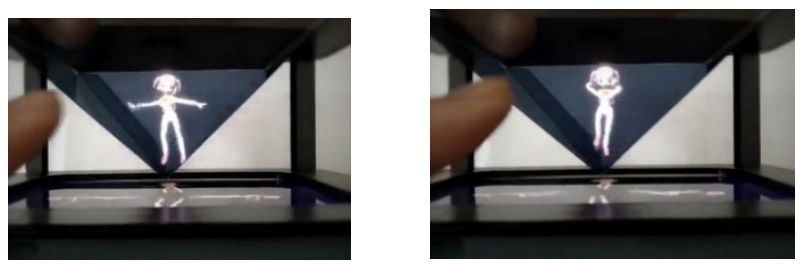

Figure 5.

Figure 5 are experimental verifications of a equipment playback source. It can play the source picture clearly and clearly, and can view the angle at any angle at 360 degrees. This shows that the family holographic projection entertainment equipment can achieve the effect of naked eye stereoscopic display.

\section{Conclusion}

Through the realization of the external three-dimensional structure of the equipment and the production of the internal chip source with the aid of holographic projection and optical principle. Then it reaches the stereoscopic display effect. The creative solution in the research has solved the problem that the moving subject cannot acquire the large image information during the previous production of the holographic chip source. Break through the technical bottleneck that the image cannot do complex 
motion and can only do the original motion around the center point. On this basis, the use of motion capture method can be more efficient action information. In addition, in the aspect of source production, it can be targeted to combine the users with high-quality output.

It is worth pointing out that holographic equipment is more motivate by low-child user group cognitive learning than traditional entertainment equipment in view of them. Users can view the source from multiple angles to get a more vivid experience. Besides, it also can be used to create atmosphere. For example, if you tell your child a story about the ocean at night, you can open the source of the underwater world's holographic video and dim the lights. Follow-up studies can be combined with other practical applications in many ways.

\section{Acknowledgments}

This work was financially supported by Study on the application of Manchu image design to promote the development of Liaoning culture tourism (Project number: W2015313).

\section{References}

1. X.Wu, W.Liao, H.G.Liu, CJL, 417-420 (2009)

2. Q.H.Wang, A.H.Wang, D.Liang, H.Deng, VE, 1-6 (2011)

3. Y.Yang, ES\&CM, 94,98 (2013)

4. Q.Wang, Scientific advisory, 64-65 (2014)

5. G.B.Fang, Q.Q.Zhu, Y.H.Shen, G.J.E.Hu, Wireless interconnected technology, 140-141(2015) 